\title{
Structure Analysis Based Parking Slot Marking Recognition for Semi-automatic Parking System
}

\author{
Ho Gi Jung ${ }^{1,2}$, Dong Suk Kim ${ }^{1}$, Pal Joo Yoon ${ }^{1}$, and Jaihie Kim ${ }^{2}$ \\ ${ }^{1}$ MANDO Corporation Central R\&D Center, Advanced Electronic System Team \\ 413-5, Gomae-Dong, Giheung-Gu, Yongin-Si, Kyonggi-Do 446-901, Republic of Korea \\ \{hgjung, greenhupa, pjyoon\} @mando.com \\ http: / / www. mando.com/eng/main.sap \\ ${ }^{2}$ Yonsei University, School of Electrical and Electronic Engineering \\ 134, Sinchon-Dong, Seodaemun-Gu, Seoul 120-749, Republic of Korea \\ \{hgjung, jhkim\}@yonsei.ac.kr \\ http://cherup.yonsei.ac.kr
}

\begin{abstract}
Semi-automatic parking system is a driver convenience system automating steering control required during parking operation. This paper proposes novel monocular-vision based target parking-slot recognition by recognizing parking-slot markings when driver designates a seed-point inside the target parking-slot with touch screen. Proposed method compensates the distortion of fisheye lens and constructs a bird's eye view image using homography. Because adjacent vehicles are projected along the outward direction from camera in the bird's eye view image, if marking line-segment distinguishing parking-slots from roadway and front-ends of marking linesegments dividing parking-slots are observed, proposed method successfully recognizes the target parking-slot marking. Directional intensity gradient, utilizing the width of marking line-segment and the direction of seed-point with respect to camera position as a prior knowledge, can detect marking linesegments irrespective of noise and illumination variation. Making efficient use of the structure of parking-slot markings in the bird's eye view image, proposed method simply recognizes the target parking-slot marking. It is validated by experiments that proposed method can successfully recognize target parkingslot under various situations and illumination conditions.
\end{abstract}

\section{Introduction}

Semi-automatic parking system is a driver convenience system automating steering control required during parking operation. Because recently driver's interest about parking assist system increases drastically, car manufacturers and component providers are developing various kinds of parking assist systems [1][2]. Fig. 1 shows the configuration of semi-automatic parking system currently being developed. The system consists of six components: EPS (Electric Power Steering) for active steering, vision sensor acquiring rear-view image, ultra-sonic sensors measuring distances to nearby side/rear obstacles, touch screen based HMI (Human Machine Interface) pro viding information to driver and receiving command from driver, EPB (Electric 
Parking Braking) automatically activating parking brake, and processing computer. Algorithms running on the processing computer consist of three components: target parking position designation, path planning generating trajectory from current position to target position, and path tracker which continuously estimates current position and controls steering system to achieve the planned path.

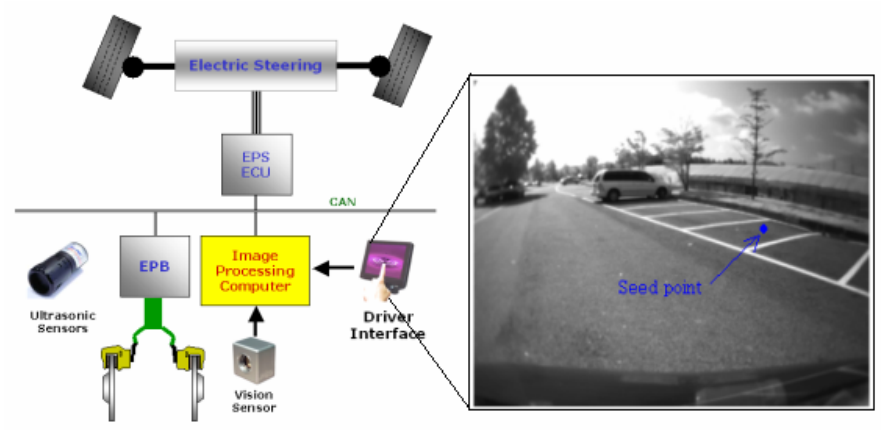

Fig. 1. System configuration of semi-automatic parking system

There are many kinds of methods for the target parking position designation: laserscanner based method [3], SRR (Short Range Radar) network based method [1], computer vision based method, GPS/digital map based method [4], and driver's manual designation based method. Prius IPAS (Intelligent Parking Assist System), mass-produced by Toyota and AISIN SEIKI in 2003, is the example of manual designation method [5]. Computer vision based method, which can provides monitoring view of ongoing parking procedure, attracts more and more interests. Computer vision based method can be categorized into three kinds: method recognizing adjacent vehicles, method recognizing parking-slot markings, and method recognizing both adjacent vehicles and parking-slot markings. Nico Kaempchen developed a system localizing free parking space by recognizing adjacent vehicles with stereo vision based method [6]. Jin Xu developed monocular vision based parking-slot marking recognition using neural network [7]. In previous research, we developed stereo vision based parking-slot marking recognition considering adjacent vehicles [8]. AISIN SEIKI's next generation is expected to recognize adjacent vehicles three-dimensionally by motion stereo and provide rendered image from a virtual viewpoint suitable for the understanding of parking operation [9].

When driver designates a seed-point inside target parking-slot with touch screen as shown in Fig. 1, proposed method recognizes corresponding parking-slot marking as target parking position. Proposed method is designed not only to solve the discomfort of previous Prius's fully manual designation method, but also to eliminate the overweighed requirements of stereo vision based method, i.e. high-performance hardware and enormous computing power. After the compensation of fisheye lens distortion and the construction of bird's eye view image, marking line-segments crossed by the gaze from camera to seed-point are detected. Guideline, distinguishing parking-slots from roadway, can be easily detected by simply finding the nearest 
among the detected line-segments. Consecutively, separating line-segments are detected based on the detected guideline. Experimental results show that proposed method is simple and robust to noise and illumination change.

\section{Bird's Eye View Construction}

Proposed system compensates the fisheye distortion of input image and constructs bird's eye view image using homography. Installed rear view camera uses fisheye lens, or wide-angle lens, to cover wide FOV (Field Of View) during parking procedure. As shown in Fig. 2, input image through fisheye lens can capture wide range of rear scene but inevitably includes severe distortion. It is well known that the major factor of fisheye lens distortion is radial distortion, which is defined in terms of the distance from the image center [10]. Modeling the radial distortion in 5th order polynomial using Caltech calibration toolbox and approximating its inverse mapping by 5 th order polynomial, proposed system acquires undistorted image as shown in Fig. 2 [11]. Homography, which defines one-to-one correspondence between coordinate in undistorted image and coordinate in bird's eye view image, can be calculated from the height and angle of camera with respect to the ground surface [8]. Bird's eye view is the virtual image taken from the sky assuming all objects are attached onto the ground surface. General pinhole camera model causes perspective distortion, by which the size of object image is changing according to the distance from camera. Contrarily, because bird's eye view image eliminates the perspective distortion of objects attached onto the ground surface, it is suitable for the recognition of objects painted on the ground surface. Final image of Fig. 2 is the bird's eye view image of the undistorted image. Hereafter, almost image processing is fulfilled in the bird's eye view image and characters in lower case bold face, e.g. u, represent coordinate or vector in the bird's eye view image.

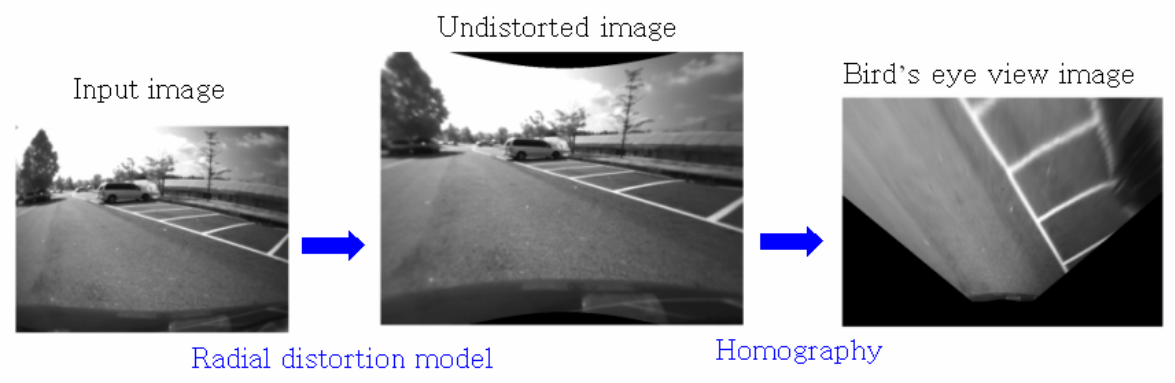

Fig. 2. Construction procedure of bird's eye view image

\section{Guideline Recognition}

Parking-slot markings consist of one guideline and separating line-segments as shown in Fig. 3(a). To recognize the parking-slot markings, marking line-segment distinguishing 
parking-slots from roadway should be recognized at first. Because the line-segment is the reference of remaining recognition procedures, it is called guideline. Each parking slot is distinguished by two line-segments perpendicular to the guideline, which is called separating line-segment.

\subsection{Marking Line-Segment Recognition by Directional Intensity Gradient}

Proposed system recognizes marking line-segments using directional intensitygradient on a line lying from seed-point to camera. As shown in Fig. 3(a), vector from seed-point to camera is represented by $\mathbf{v}_{\text {seed point-camera }}$ and its unit vector is represented

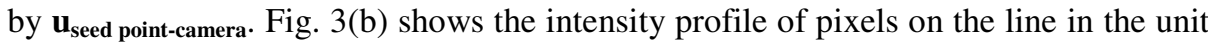
of pixel length s. If the start point $\mathbf{p}_{\mathbf{s}}$ and unit vector $\mathbf{u}$ are fixed, the intensity of a pixel which is distant by $\mathrm{s}$ in the direction of $\mathbf{u}$ from $\mathbf{p}_{\mathbf{s}}$, i.e. $\mathrm{I}\left(\mathbf{p}_{\mathbf{s}}+\mathrm{s}^{\cdot} \mathbf{u}\right)$, is represented by simple notation I(s). Because the line crosses two line-segments, it can be observed that two intensity peaks with the width of ling-segment exist.

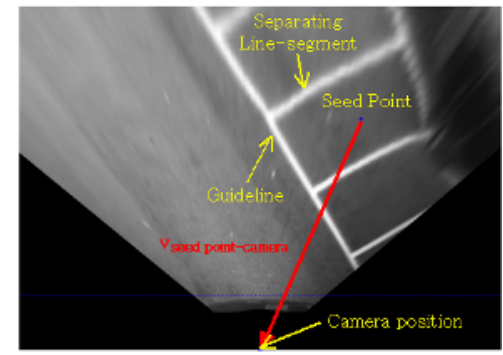

(a) $\mathbf{v}_{\text {seed point-camera }}$

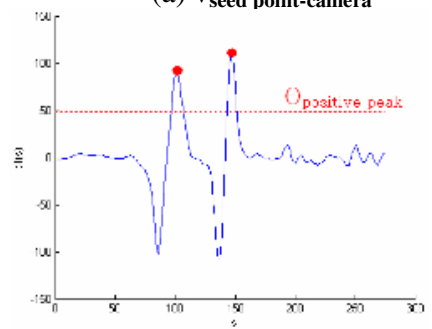

(c) $\mathrm{dI}(\mathrm{s})$ and recognized edges

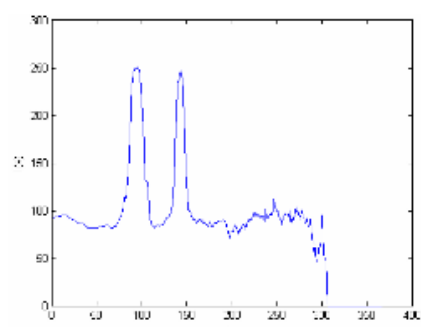

(b) Intensity profile

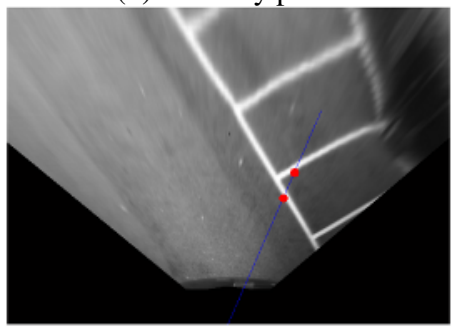

(d) recognized marking line-segments

Fig. 3. Procedure of marking line-segment recognition

Equation (1) defines the directional intensity-gradient of a point $\mathbf{p}(\mathrm{x}, \mathrm{y})$ with respect to vector $\mathbf{u}, \mathrm{dI}(\mathbf{p}, \mathbf{u})$. Because camera maintains a certain height and angle with respect to the ground surface, marking line-segment painted on the ground surface will appear with a fixed width $\mathrm{W}$. Therefore, directional intensity-gradient using the average intensity of W/2 interval is robust to noise while detecting interesting edges.

$$
d I(\mathbf{p}, \mathbf{u})=\frac{1}{\frac{W}{2}} \sum_{i=1}^{\frac{W}{2}} I(\mathbf{p}-i \cdot \mathbf{u})-\frac{1}{\frac{W}{2}} \sum_{i=1}^{\frac{W}{2}} I(\mathbf{p}+i \cdot \mathbf{u})
$$


Fig. 3(c) shows the profile of directional intensity-gradient of the line with respect to $\mathbf{u}_{\text {seed point-camera }}$, i.e. $\mathrm{dI}\left(\mathbf{p}_{\text {seed point }}+\mathrm{s}^{*} \mathbf{u}_{\text {seed point-camera }}, \mathbf{u}_{\text {seed point-camera }}\right)$, which is denoted by simple notation dI(s). Positive peaks correspond to the camera-side edges of marking line-segments and negative peaks correspond to the seed-point-side edges. Because camera-side edge is easy to follow, it is recognized as the position of marking line-segment. Threshold for positive peak detection, $\theta_{\text {positive peak }}$, is defined adaptively like equation (2). Fig. 3(c) shows established threshold and recognized positive peaks. Fig. 3 (d) shows the recognized marking line-segments in bird's eye view image.

$$
\theta_{\text {positi ve peak }}=\frac{1}{3}\left(\max _{\mathrm{s}} I(s)-\underset{s}{\operatorname{avg} I(s)}\right)
$$

\subsection{Recognition of Marking Line-Segment Direction}

Proposed system detects the direction of marking line-segments using the directional intensity-gradient of local window and edge following based refinement. Edge following results can eliminate falsely detected marking line-segments.

The directional intensity-gradient of a point displaced by $(\mathrm{dx}, \mathrm{dy})$ from a center point $\mathbf{p}_{\mathbf{c}}\left(\mathrm{x}_{\mathrm{c}}, \mathrm{y}_{\mathrm{c}}\right)$ can be calculated by $\mathrm{dI}\left(\mathbf{p}_{\mathrm{c}}+(\mathrm{dx}, \mathrm{dy}), \mathbf{u}\right)$, which is denoted by simple notation $\mathrm{dI}(\mathrm{dx}, \mathrm{dy})$ if $\mathbf{p}_{\mathbf{c}}$ and $\mathbf{u}$ are fixed. Proposed system calculates the directional intensity-gradient, $\mathrm{dI}\left(\mathbf{p}_{\text {cross }}+(\mathrm{dx}, \mathrm{dy}), \mathbf{u}_{\text {seed point-camera }}\right)$, of $(\mathrm{W}+1) \mathrm{x}(\mathrm{W}+1)$ local window around the detected cross-points $\mathbf{p}_{\text {cross. }}$. Here, $d x$ and dy are in the range of $-W / 2 \sim W / 2$. Fig. 4 shows the calculated $\mathrm{dI}(\mathrm{dx}, \mathrm{dy})$ of local window around a cross-point. It can be observed that $\mathrm{dI}(\mathrm{dx}, \mathrm{dy})$ array forms a ridge, of which direction is the same as the edge direction.

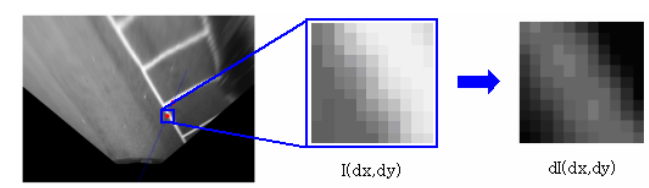

Fig. 4. Directional intensity-gradient of local window around a cross-point

To detect the direction of the ridge, proposed system introduces fitness ridge $(\phi)$, which measures how well a line rotating by $\phi$ around the cross-point is similar to the ridge direction like equation (3). As shown in Fig. 5(a), fitness ridge $_{\phi} \phi$ ) is the difference between two line-sums in $\mathrm{dI}(\mathrm{dx}, \mathrm{dy})$. These lines are orthogonal to each other.

$$
\text { fitness }_{\text {ridge }}(\phi)=\sum_{\mathrm{i}=-\frac{\mathrm{W}}{2}}^{\frac{\mathrm{W}}{2}} d I(i \cdot \cos (\phi), i \cdot \sin (\phi))-\sum_{\mathrm{i}=-\frac{\mathrm{W}}{2}}^{\frac{\mathrm{W}}{2}} d I\left(i \cdot \cos \left(\phi+\frac{\pi}{2}\right), i \cdot \sin \left(\phi+\frac{\pi}{2}\right)\right)
$$

Fig. 5(b) shows calculated fitness ridge $(\phi)$ in the range of $0 \sim 180^{\circ}$ and it can be approximated by a cosine function whose frequency $\mathrm{f}_{0}$ is $1 / 180^{\circ}$ like equation (4). 
To eliminate the effect of noise, estimated phase parameter is used to estimate the ridge direction like equation (5). In general, amplitude and phase parameter can be estimated by MLE (Maximum Likelihood Estimation) [12]. Estimated cosine function in Fig. 5(b) shows that the maximum value of fitness ridge $(\phi)$ can be robustly detected.

$$
\text { fitness }_{\text {ridge }}[n]=A \cdot \cos \left(2 \pi f_{0} n+\psi\right)+w[n]
$$

where, $n$ : integer index of $\phi, w[n]$ : white gausian noise

$$
\phi_{\text {ridge }}=-\frac{\hat{\psi}}{2 \pi f_{0}}, \text { where } \hat{\psi}=\tan ^{-1}\left(\frac{-\sum_{n=0}^{179} \text { fitness }_{\text {ridge }}[n] \cdot \sin \left(2 \pi f_{0} n\right)}{\sum_{n=0}^{179} \text { fitness }_{\text {ridge }}[n] \cdot \cos \left(2 \pi f_{0} n\right)}\right)
$$

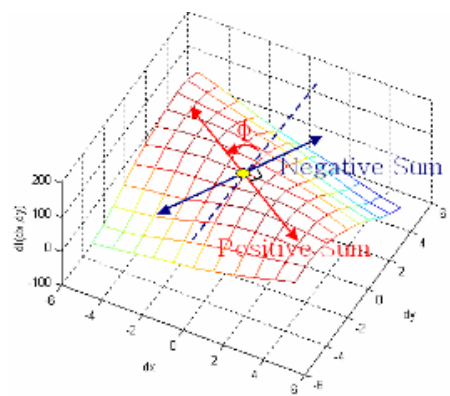

(a) $\mathrm{dI}(\mathrm{dx}, \mathrm{dy})$ in $3 \mathrm{D}$ display

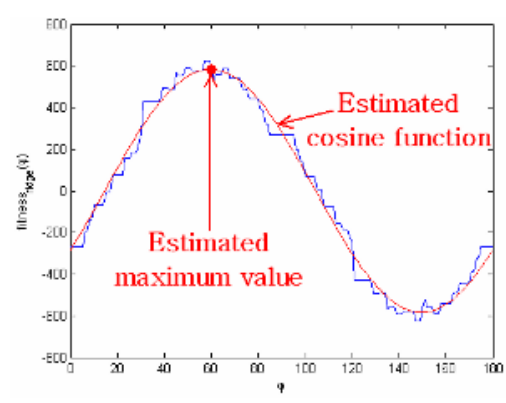

(b) Estimated maximum value of fitness ridge $_{\text {in }}(\phi)$

Fig. 5. Estimation of line-segment direction by model based fitness estimation

Edge following, starting from the detected cross-point in the estimated edge direction, refines the edge direction and eliminates falsely detected cross-points. Edge position estimate of $n+1$ step can be calculated by cross-point $\mathbf{p}_{\text {edge }}[0]$ and edge direction of $\mathrm{n}$ step $\mathbf{u}_{\text {edge }}[\mathrm{n}]$ like equation (6). Finding maximum of local directional intensity-gradient $\mathrm{dI}(\mathrm{t})$, defined like equation (7), updates the edge position of $\mathrm{n}+1$ step like equation (8). $\mathbf{n}_{\text {edge }}[n]$ is the unit vector normal to $\mathbf{u}_{\text {edge }}[n]$ and $t_{\max }[n]$ is the relative position maximizing $\mathrm{dI}(\mathrm{t})$ in $\mathbf{n}_{\text {edge }}[\mathrm{n}]$ direction as shown in Fig. 6(a). Iterating edge following terminates if new edge strength $\mathrm{dI}\left(\mathrm{t}_{\max }[\mathrm{n}+1]\right)$ is definitely smaller than the edge strength of cross point $\mathrm{dI}\left(\mathrm{t}_{\max }[0]\right)$, e.g. $70 \%$. Proposed system rejects detected cross-points of which successful edge following iteration is smaller than a certain threshold $\theta$ edge following to eliminate falsely detected marking line-segments. Consequently, refined edge direction $\mathbf{u}_{\text {edge }}[n+1]$ is set to a unit vector from $\mathbf{p}_{\text {edge }}[0]$ to $\mathbf{p}_{\text {edge }}[\mathrm{n}+1]$. Fig. 6(b) shows the edge following results and refined direction.

$$
\begin{gathered}
\hat{\mathbf{p}}_{\text {edge }}[n+1]=\mathbf{p}_{\text {edge }}[0]+(n+1) \cdot d s \cdot \mathbf{u}_{\text {edge }}[n] \\
d I(t)=d I\left(\hat{\mathbf{p}}_{\text {edge }}[n+1]+t \cdot \mathbf{n}_{\text {edge }}[n], \mathbf{n}_{\text {edge }}[n]\right), \text { where } t:-\frac{W}{2} \sim \frac{W}{2}
\end{gathered}
$$




$$
\mathbf{p}_{\text {edge }}[n+1]=\hat{\mathbf{p}}_{\text {edge }}[n+1]+t_{\max }[n+1] \cdot \mathbf{n}_{\text {edge }}[n]
$$

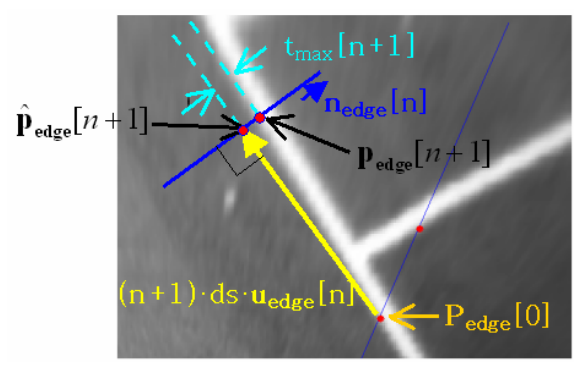

(a) Edge following method

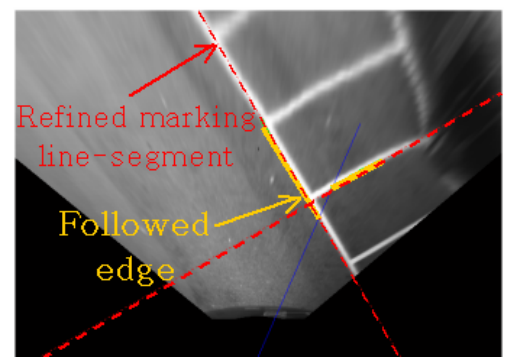

(b) Edge following results

Fig. 6. Edge following refines the direction of detected marking line-segments

\subsection{Determination of Guideline}

If the seed-point designated by driver is locating in a valid parking-slot, gaze-line from seed-point to camera should meet marking line-segments more than once making corresponding cross-points. Among marking line-segments validated by the edge following, guideline is the marking line-segment of which cross-point is nearest to the camera position. In other words, guideline has smallest distance between crosspoint and camera, i.e. $\left|\mathbf{p}_{\text {camera }}-\mathbf{p}_{\text {edge }}[0]\right|$. Fig. 7 shows recognized guideline.

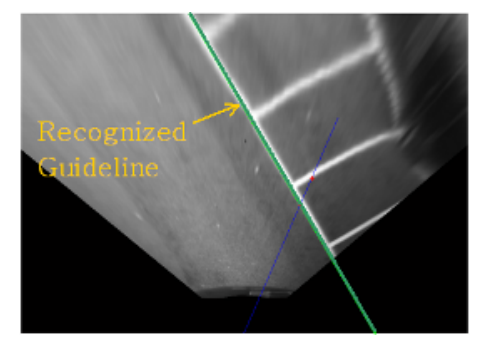

Fig. 7. Guideline recognized by structural relation between cross-points

\section{Recognition of Target Parking-Slot}

By searching separating line-segments bi-directionally from selection point $\mathbf{p}_{\text {selection }}$ that is obtained by projecting the seed-point onto the guideline, proposed system recognizes the exact location of target parking-slot. $\mathbf{p}_{\text {selection }}$ is calculated by crosspoint $\mathbf{p}_{\text {cross }}$ and guideline unit vector $\mathbf{u}_{\text {guideline }}$ like equation (9).

$$
\mathbf{p}_{\text {selection }}=\mathbf{p}_{\text {cross }}+\left(\mathbf{u}_{\text {guideline }} \cdot\left(\mathbf{p}_{\text {seed point }}-\mathbf{p}_{\text {cross }}\right)\right) \mathbf{u}_{\text {guideline }}
$$




$$
\begin{gathered}
I_{o n}(s)=\frac{1}{\frac{W}{2}} \sum_{t=0}^{\frac{W}{2}} I\left(\mathbf{p}_{\text {selection }}+s \cdot \mathbf{u}_{\text {searching }}+t \cdot \mathbf{n}_{\text {guideline }}\right) \\
I_{\text {off }}(s)=\frac{1}{\frac{W}{2}} \sum_{t=\frac{3}{2} W}^{2 W} I\left(\mathbf{p}_{\text {selection }}+s \cdot \mathbf{u}_{\text {searching }}+t \cdot \mathbf{n}_{\text {guideline }}\right)
\end{gathered}
$$

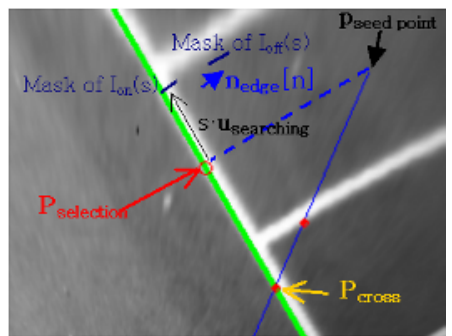

(a) Mask for $\mathrm{I}_{\text {on }}(\mathrm{s})$ and $\mathrm{I}_{\text {off }}(\mathrm{s})$

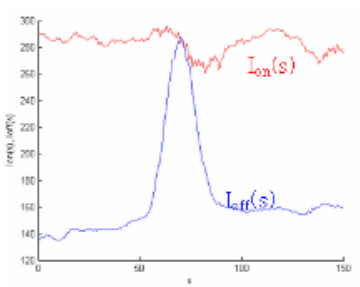

(b) in $\mathbf{u}_{\text {guideline }}$ direction

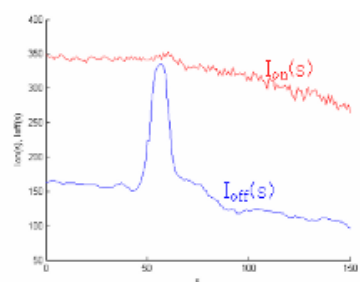

(c) in $-\mathbf{u}_{\text {guideline }}$ direction

Fig. 8. Measuring $I_{o n}(s)$ and $I_{\text {off }}(s)$ bi-directionally to find both-side ' $T$ '-shape junctions

Searching ' $\mathrm{T}$ ' -shape junction between guideline and separating line-segment can detect the position of the separating line-segment. Average intensity on the guideline marking, $\mathrm{I}_{\mathrm{on}}(\mathrm{s})$, is measured like equation (10) and the average intensity of neighboring region outward from camera, $\mathrm{I}_{\mathrm{off}}(\mathrm{s})$, is measured like equation (11). Here, $\mathbf{u}_{\text {searching }}$ is either $\mathbf{u}_{\text {guideline }}$ or $-\mathbf{u}_{\text {guideline }}$ according to the search direction. Fig. 8(a) depicts the procedure of measuring $\mathrm{I}_{\text {on }}(\mathrm{s})$ and $\mathrm{I}_{\text {off }}(\mathrm{s})$. Fig. 8(b) and (c) shows the measured $\mathrm{I}_{\text {on }}(\mathrm{s})$ and $\mathrm{I}_{\text {off }}(\mathrm{s})$ in both directions. It can be observed that $\mathrm{I}_{\text {off }}(\mathrm{s})$ is similar to $\mathrm{I}_{\mathrm{on}}(\mathrm{s})$ only around ' $\mathrm{T}$ '-shape junction. Therefore, the location of junction can be detected by thresholding the ratio of $\mathrm{I}_{\text {off }}(\mathrm{s})$ to $\mathrm{I}_{\mathrm{on}}(\mathrm{s})$, named $\mathrm{L}_{\text {separating }}(\mathrm{s})$. Fig. 9 (a) and (b) shows detected junction and Fig. 9(c) shows recognized target parking-slot.

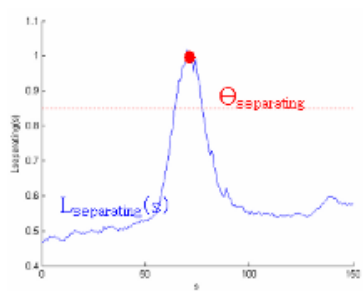

(a) in $\mathbf{u}_{\text {guideline }}$ direction

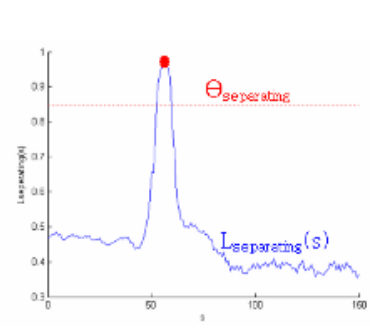

(b) in $-\mathbf{u}_{\text {guideline }}$ direction

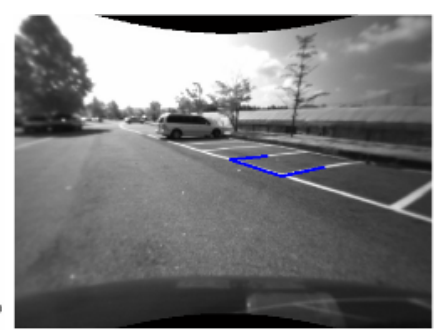

(c) Recognized target

Fig. 9. $\mathrm{L}_{\text {separating }}(\mathrm{s})$ can find separating line-segments irrespective of local intensity variation

\section{Experimental Results and Conclusion}

In bird's eye view image, objects above the ground surface are projected outward from camera. Therefore, only if guideline and the ' $T$ '-shape junctions of target parking-slot are observed, proposed method can successfully detect cross-points, 


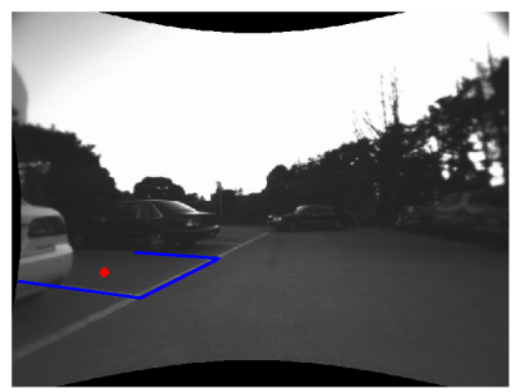

(a) Recognized target parking-slot

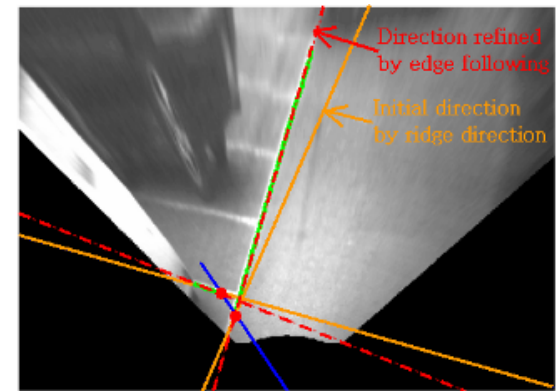

(b) Direction refinement by edge following

Fig. 10. Case with adjacent vehicles and torn markings

related marking line-segments and separating line-segments as shown in Fig. 10. Fig 10(a) is captured against the light and markings are torn to be noisy. In Fig. 10(b), edge following based edge direction refinement overcomes the error of initial direction estimation.

Separating line-segment detection method considering locally changing illumination condition can successfully detect target parking-slot even if local intensities are different from each other. Fig. 11 shows that $\mathrm{L}_{\text {separating }}(\mathrm{s})$ can compensate local intensity variation.

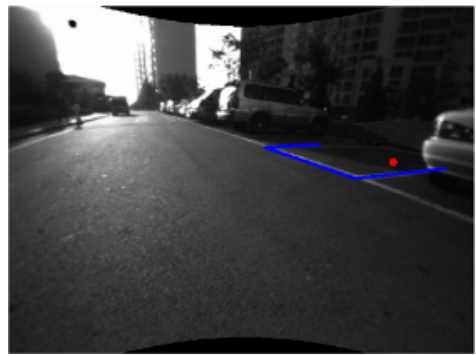

(a) Recognized target parking-slot

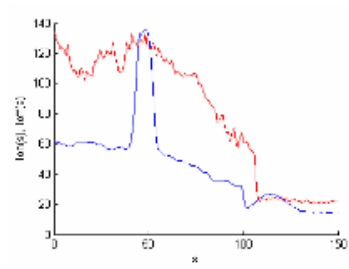

(b) $\mathrm{I}_{\text {on }}(\mathrm{s})$ and $\mathrm{I}_{\text {off }}(\mathrm{s})$

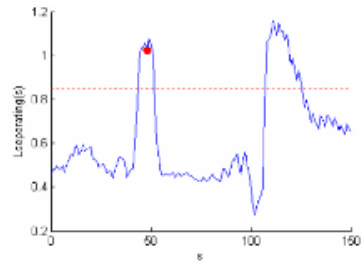

(c) $\mathrm{L}_{\text {separating }}(\mathrm{s})$

Fig. 11. Case with strong sunshine causing locally changing intensity

Major contribution of this paper is that because parked vehicles are projected outward from camera in bird's eye view image, if guideline and ' $T$ '-shape junctions are observed, guideline can be detected simply by finding the cross-point nearest from camera. Because proposed method uses small portion of input image and fully utilizes structural characteristics of parking-slot markings in bird's eye view image, it can achieve concise implementation and deterministic performance.

\section{References}

1. Richard Bishop: Intelligent Vehicle Technology and Trends, Artech House Pub. (2005)

2. Randy Frank: Sensing in the Ultimately Safe Vehicle, SAE Paper No.: 2004-21-0055, Society of Automotive Engineers (2004) 
3. Alexander Schanz, et al.: Autonomous Parking in Subterranean Garages - A Look at the Position Estimation, IEEE Intelligent Vehicle Symposium (2003), 253-258

4. Massaki Wada, et al.: Development of Advanced Parking Assistance System, IEEE Trans. Industrial Electronics, Vol. 50, No. 1 (2003), 4-17

5. Masayuki Furutani: Obstacle Detection Systems for Vehicle Safety, SAE Paper No.: 200421-0057, Society of Automotive Engineers (2004)

6. Nico Kaempchen, et al.: Stereo Vision Based Estimation of Parking Lots Using 3D Vehicle Models, IEEE Intelligent Vehicle Symposium (2002), 459-464 vol.2

7. Jin $\mathrm{Xu}$, et al.: Vision-Guided Automatic Parking for Smart Car, IEEE Intelligent Vehicle Symposium (2000), 725-730

8. Ho Gi Jung, et al.: Stereo Vision Based Localization of Free Parking Site, CAIP 2005, LNCS 3691 (2005), 231-239

9. C. Vestri, et al.: Evaluation of a Vision-Based Parking Assistance System, IEEE Conf. Intelligent Transportation Systems (2005), 56-60

10. J. Salvi, et al.: A Comparative Review of Camera Calibrating Methods with Accuracy Evaluation, Pattern Recognition 35 (2002), 1617-1635

11. J. Y. Bouguet: Camera Calibration Toolbox for Matlab, http://www.vision.caltech.edu/ bouguetj/calib_doc/index.html

12. Steven M. Key: Fundamentals of Statistical Signal Processing, Volume I: Estimation Theory, Prentice Hall Inc. (1993), 193-195 